\title{
SOME INTERDEPENDENCIES OF SUM AND PRODUCT INTEGRALS
}

\author{
JON C. HELTON
}

\begin{abstract}
In a recent paper Davis and Chatfield show if $\int_{a}^{b} G^{2}=0$, then $\int_{a}^{b} G$ exists if and only if $\prod_{a}^{b}(1+G)$ exists and is not zero. In this paper we extend that result and prove if $\beta>0,|G|<$ $1-\beta$ on $[a, b]$ and $\int_{a}^{b} G^{2}$ exists, then $\int_{a}^{b} G$ exists if and only if $\prod_{a}^{b}(1+G)$ exists and is not zero. Furthermore, if $\prod_{x}^{y}(1+G)=$ $\exp \int_{x}^{y} G$ for $a \leqq x<y \leqq b$, then $\int_{a}^{b} G^{2}=0$.
\end{abstract}

All integrals and definitions are of the subdivision-refinement type, $R$ denotes the set of all real numbers, and functions are $R$-valued and understood to be defined only on $\{x, y\}$ in $R \times R$ such that $x<y$. The statement that $G$ is bounded, $G \in O P^{\circ}$ or $G \in O B^{\circ}$ on $[a, b]$ means there exists a subdivision $D$ of $[a, b]$ and a number $B$ such that if $\left\{x_{q}\right\}_{q=0}^{n}$ is a refinement of $D$, then

(1) if $0<q \leqq n$, then $\left|G_{q}\right|<B$,

(2) if $0<u \leqq v \leqq n$, then $\left|\prod_{q=u}^{v}\left(1+G_{q}\right)\right|<B$, or

(3) $\sum_{q=1}^{n}\left|G_{q}\right|<B$,

respectively, where $G_{q}=G\left(x_{q-1}, x_{q}\right)$. Similarly, statements of the form $G>b$ should be interpreted in terms of subdivisions and refinements. The sequence of functions $\left\{S_{n}\right\}_{n=1}^{\infty}$ converges uniformly to $S$ on $[a, b]$ means if $\varepsilon>0$ then there exists a subdivision $D$ of $[a, b]$ and a positive integer $N$ such that if $\left\{x_{q}\right\}_{q=0}^{r}$ is a refinement of $D, n>N$, and $0<q \leqq r$ then $\left|S_{n}\left(x_{q-1}, x_{q}\right)-S\left(x_{q-1}, x_{q}\right)\right|<\varepsilon$. Further, $G \in O L^{\circ}$ on $[a, b]$ if and only if $\lim _{x \rightarrow p^{-}} G(x, p), \lim _{x \rightarrow p^{+}} G(p, x), \lim _{x, y \rightarrow p^{-}} G(x, y)$ and $\lim _{x, y \rightarrow p^{+}} G(x, y)$ exist for $p \in[a, b]$. Also, $G \in O A^{\circ}$ or $G \in O M^{\circ}$ on $[a, b]$ if and only if $\int_{a}^{b} G$ exists and $\int_{a}^{b}\left|G-\int G\right|=0$ or $\prod_{x}^{y}(1+G)$ exists for $a \leqq x<y \leqq b$ and $\int_{a}^{b}|1+G-\Pi(1+G)|=0$, respectively. For $R$-valued functions, the class $O A^{\circ}$ on $[a, b]$ is the class of functions $G$ such that $\int_{a}^{b} G$ exists $[4$, p. 669]. The sources of these definitions are found in [2, p. 299] and [3, p. 493].

THEOREM 1. If $G \in O B^{\circ}$ on $[a, b],\left\{S_{n}\right\}_{n=1}^{\infty}$ is a sequence of functions converging uniformly to $S$ on $[a, b]$ and $\int_{a}^{b} S_{n} G$ exists for $n=1,2,3, \cdots$, then $\int_{a}^{b} S G$ exists, $\lim _{n \rightarrow \infty} \int_{a}^{b} S_{n} G$ exists, and $\int_{a}^{b} S G=\lim _{n \rightarrow \infty} \int_{a}^{b} S_{n} G$.

The proof of Theorem 1 is omitted.

Presented to the Society, September 2, 1971; received by the editors October 3, 1971 and, in revised form, May 17, 1972.

AMS (MOS) subject classifications (1970). Primary 26A39, 26 A42.

Key words and phrases. Sum integral, product integral, interdependency, exponential, bound, interval function.

(c) American Mathematical Society 1973 
LEMMA 1. If $\prod_{a}^{b}(1+G)$ exists and is not zero and $p \in(a, b]$, then there exists $\delta>0$ such that if $p-\delta<x<p$, then $\mid G(x, p)-G(x, y)-G(y, p)-$ $G(x, y) G(y, p) \mid$ is bounded as $y \rightarrow p^{-}$. If, furthermore, $G(y, p)$ is bounded as $y \rightarrow p^{-}$, then

$$
\lim _{x, y \rightarrow \nu^{-}}[G(x, p)-G(x, y)-G(y, p)-G(x, y) G(y, p)]=0 .
$$

LeMmA 2. If $\int_{a}^{b} G$ exists and $p \in(a, b]$, then

$$
\lim _{x . y \rightarrow p^{-}}[G(x, y)+G(y, p)-G(x, p)]=0 .
$$

Lemmas 1 and 2 can be established by using the Cauchy criteria for product and sum integrals, respectively.

THEOREM 2. If $\prod_{a}^{b}(1+G)$ exists and is not zero and $\int_{a}^{b} G^{2}$ exists, then $G \in O L^{\circ}$ on $[a, b]$.

Proof. Since $\int_{a}^{b} G^{2}$ exists, it follows

(1) if $p \in(a, b]$, then $\lim _{x, y \rightarrow p^{-}} G(x, y)=0$, and

(2) if $p \in[a, b)$, then $\lim _{x, y \rightarrow p^{+}} G(x, y)=0$, and hence it is only necessary to show that

(3) if $p \in(a, b]$, then $\lim _{x \rightarrow p^{-}} G(x, p)$ exists, and

(4) if $p \in[a, b)$, then $\lim _{x \rightarrow p^{+}} G(p, x)$ exists.

Let $p \in(a, b]$. Thus, since $(1)$ is true and $G(y, p)$ is bounded as $y \rightarrow p^{-}$, it follows from Lemma 1 that (3) is true. In a similar manner, statement (4) can be shown to be true; therefore, $G \in O L^{\circ}$ on $[a, b]$.

THEOREM 3. If $\int_{a}^{b} G$ exists and $\int_{a}^{b} G^{2}$ exists, then $G \in O L^{\circ}$ on $[a, b]$.

The proof is similar to the proof of Theorem 2 .

THEOREM 4. If $-1<G$ on $[a, b]$, then the following statements are equivalent :

(1) $\prod_{a}^{b}(1+G)$ exists and is not zero, and

(2) $\int_{a}^{b} \ln (1+G)$ exists.

Furthermore, $\prod_{a}^{b}(1+G)=\exp \int_{a}^{b} \ln (1+G)$.

IndiCATION OF PROOF. (1) $\rightarrow(2)$.

$$
\begin{aligned}
\left|\ln \prod_{a}^{b}(1+G)-\sum_{H} \ln \left(1+G_{q}\right)\right| & =\left|\ln \left[\alpha \cdot \prod_{H}\left(1+G_{a}\right)\right]-\sum_{H} \ln \left(1+G_{a}\right)\right| \\
& =|\ln \alpha| \rightarrow 0 .
\end{aligned}
$$


INDICATION OF PROOF. (2) $\rightarrow(1)$.

$$
\begin{aligned}
\mid \exp \int_{a}^{b} \ln (1+G)- & \prod_{H}\left(1+G_{q}\right) \mid \\
& =\left|\exp \left[\alpha+\sum_{H} \ln \left(1+G_{q}\right)\right]-\prod_{H}\left(1+G_{q}\right)\right| \\
& =\left|\left(e^{\alpha}-1\right) \prod_{H}\left(1+G_{q}\right)\right| \rightarrow 0 .
\end{aligned}
$$

THEOREM 5. If $\beta>0,|G|<1-\beta$ on $[a, b]$ and $\int_{a}^{b} G^{2}$ exists, then the following statements are equivalent:

(1) $\prod_{a}^{b}(1+G)$ exists and is not zero, and

(2) $\int_{a}^{b} G$ exists.

Proof. (1) $\rightarrow(2)$. Note that $G^{2} \in O A^{\circ}$ and $O B^{\circ}$ on $[a, b]$. It follows from Theorem 2 that $G \in O L^{\circ}$ on $[a, b]$. Hence, $\sum_{n=2}^{p}(-1)^{n-1}\left[G^{n-2}\right] / n \in O L^{\circ}$ on $[a, b]$ for $p=2,3,4, \cdots$. Thus, $\int_{a}^{b}\left\{\sum_{n=2}^{p}(-1)^{n-1}\left[G^{n-2}\right] / n\right\} G^{2}$ exists for $p=2,3,4, \cdots[3$, Theorem 2 , p. 494]. Therefore, since

$$
\left\{\sum_{n=2}^{p}(-1)^{n-1}\left[G^{n-2}\right] / n\right\}_{p=2}^{\infty}
$$

converges uniformly to $\sum_{n=2}^{\infty}(-1)^{n-1}\left[G^{n-2}\right] / n$ on $[a, b]$, it follows from Theorem 1 that

$$
\int_{a}^{b}\left\{\sum_{n=2}^{\infty}(-1)^{n-1}\left[G^{n-2}\right] / n\right\} G^{2}=\int_{a}^{b} \sum_{n=2}^{\infty}(-1)^{n-1} G^{n} / n
$$

exists. Further, Theorem 4 implies that

$$
\int_{a}^{b} \ln (1+G)=\int_{a}^{b} \sum_{n=1}^{\infty}(-1)^{n-1} G^{n} / n
$$

exists. Hence, $\int_{a}^{b} G$ exists.

Proof. (2) $\rightarrow(1)$. By the use of Theorems 1 and 3, it follows as in the preceding paragraph that $\int_{a}^{b} \sum_{n=2}^{\infty}(-1)^{n-1} G^{n} / n$ exists. Thus,

$$
\int_{a}^{b} \sum_{n=1}^{\infty}(-1)^{n-1} G^{n} / n=\int_{a}^{b} \ln (1+G)
$$

exists. Therefore, Theorem 4 implies that $\prod_{a}^{b}(1+G)$ exists and is not zero.

THEOREM 6. If $\prod_{a}^{b}(1+G)$ exists and is not zero and $\int_{a}^{b} G$ exists, then $G$ is bounded on $[a, b]$. 
Proof. We will show if $p \in(a, b]$ then $G(y, p)$ is bounded as $y \rightarrow p^{-}$ and $G(x, y)$ is bounded as $x, y \rightarrow p^{-}$and if $p \in[a, b)$ then $G(p, y)$ is bounded as $y \rightarrow p^{+}$and $G(x, y)$ is bounded as $x, y \rightarrow p^{+}$. Boundedness then follows by using the Heine-Borel theorem. Suppose there exists $p \in(a, b]$ such that $G(y, p)$ is unbounded as $y \rightarrow p^{-}$. Hence, there exists a sequence $\left\{y_{a}\right\}_{a=1}^{\infty}$ converging to $p$ such that $y_{q}<y_{q+1}<p$ and $G\left(y_{q}, p\right)>q$. It follows from Lemmas 1 and 2 that there exist numbers $x$ and $B$ such that if $x<y_{q}<p$, then

(1) $\left|G(x, p)-G\left(x, y_{q}\right)-G\left(y_{q}, p\right)\right|<B$, and

(2) $\left|G(x, p)-G\left(x, y_{q}\right)-G\left(y_{q}, p\right)-G\left(x, y_{q}\right) G\left(y_{q}, p\right)\right|<B$.

Hence, $G\left(x, y_{q}\right) G\left(y_{q}, p\right)$ is bounded as $q \rightarrow \infty$. Thus, $\left\{G\left(x, y_{q}\right)\right\}_{q=1}^{\infty}$ must converge to zero, and as a result, $G(x, p)-G\left(x, y_{q}\right)-G\left(y_{q}, p\right)$ is unbounded as $q \rightarrow \infty$. This contradicts (1), and therefore, $G(y, p)$ is bounded as $y \rightarrow p^{-}$. Now, if $p \in(a, b]$, it follows from Lemma 2 that $G(x, y)$ is bounded as $x, y \rightarrow p^{-}$. Boundedness follows in a similar manner for the right limits of $G$.

Therefore, it follows from the Heine-Borel theorem that there exists a number $B$ and a subdivision $\left\{x_{q}\right\}_{q=0}^{n}$ of $[a, b]$ such that if $x_{q-1} \leqq x<y \leqq x_{q}$ then $|G(x, y)|<B$.

LEMMA 3. If $G$ is bounded on $[a, b]$ and $\int_{a}^{b} G$ exists, then there exists $a$ number $B$ such that if $a \leqq x<y \leqq b$ then $\left|\int_{x}^{y} G\right|<B$.

Since $G \in O A^{\circ}$ on $[a, b]$, the proof follows easily.

THEOREM 7. If $\prod_{a}^{b}(1+G)$ exists and is not zero, then the following statements are equivalent:

(1) if $a \leqq x<y \leqq b$, then $\int_{x}^{y} G$ exists and $\prod_{x}^{y}(1+G)=\exp \int_{x}^{y} G$, and

(2) $\int_{a}^{b} G^{2}=0$.

Proof. Davis and Chatfield [1, Theorem 3, p. 744] show that (2) implies (1). Hence, we only need to show that (1) implies (2). We will do this by showing that

$$
\int_{a}^{b}\left|\sum_{n=2}^{\infty} G^{n} / n !\right|=0 .
$$

From this, the desired result follows.

Let $\varepsilon>0$. Since $G$ is bounded (Theorem 6 ), $\int_{x}^{y} G$ is bounded on $[a, b]$ (Lemma 3). Thus, $\Pi_{x}^{y}(1+G)$ and $\left[\Pi_{x}^{y}(1+G)\right]^{-1}$ are bounded for $a \leqq x<y \leqq b$. Therefore, $G \in O M^{\circ}$ on $[a, b]$ [2, Theorem 4.2, p. 305]. Also, $G \in O A^{\circ}$ on $[a, b]$, Thus, there exists a subdivision $D$ of $[a, b]$ and a number $B$ such that if $H=\left\{x_{q}\right\}_{q=0}^{n}$ is a refinement of $D$, then

(1) $\sum_{H}\left|1+G_{q}-\prod_{x_{q-1}}^{x_{q}}(1+G)\right|<\varepsilon / 2$,

(2) $\left|\exp G_{q}\right|<B$ for $x_{q-1}, x_{q} \in H$, and

(3) $\sum_{H}\left|-1+\exp \left[-G_{q}+\int_{x_{q-1}}^{x_{q}} G\right]\right|<\varepsilon / 2 B$. 
Let $H=\left\{x_{a}\right\}_{a=0}^{n}$ be a refinement of $D$, then

$$
\begin{aligned}
\varepsilon / 2 & >\sum_{H}\left|1+G_{q}-\prod \prod_{x_{q}-1}^{x_{a}}(1+G)\right| \\
& =\sum_{H}\left|1+G_{q}-\exp \int_{x_{q}-1}^{x_{q}} G\right| \\
& \left.=\sum_{H}\left|1+G_{q}-\left\{\exp G_{q}\right\}\right| 1-1+\exp \left[-G_{q}+\int_{x_{q-1}}^{x_{a}} G\right]\right\} \mid \\
& \geqq \sum_{H}\left|1+G_{q}-\exp G_{q}\right|-\sum_{H}\left|\exp G_{q}\right|\left|-1+\exp \left[-G_{a}+\int_{x_{q}-1}^{x_{a}} G\right]\right| \\
& >\sum^{H}\left|1+G_{q}-\exp G_{q}\right|-B \varepsilon / 2 B,
\end{aligned}
$$

and thus

$$
\varepsilon>\sum_{H}\left|1+G_{q}-\exp G_{q}\right|=\sum_{H}\left|\sum_{n=2}^{\infty}\left[G_{q}^{n}\right] / n !\right| .
$$

Since $\int_{a}^{b}\left|e^{G}-1-G\right|=0$ and $e^{x}-1-x$ has the minimum value of 0 at $x=0$,

$$
\sum_{n=2}^{\infty}\left[G_{a}^{n-2}\right] / n !=\frac{1}{2}+\sum_{n=3}^{\infty}\left[G_{a}^{n-2}\right] / n !>\frac{1}{4}
$$

for refinements of a suitable subdivision of $[a, b]$. Therefore, since

$$
0=\int_{a}^{b}\left|\sum_{n=2}^{\infty} G^{n} / n !\right|=\int_{a}^{b}\left|G^{2}\right|\left|\sum_{n=2}^{\infty}\left[G^{n-2}\right] / n !\right|
$$

it follows that $\int_{a}^{b} G^{2}=0$.

A special case of Theorem 7 is proved by J. A. Chatfield [Theorem 8 in "Equivalence of integrals," Proc. Amer. Math. Soc. (submitted)]. He shows that Theorem 7 is true for functions from $R \times R$ to the nonnegative numbers. However, his proof does not hold for functions from $R \times R$ to $R$.

Davis and Chatfield $[1$, p. 747] show that there exists a function $G$ such that $\int_{a}^{b} G^{2}=0, \int_{a}^{b} G$ exists and $G \notin O B^{\circ}$ on $[a, b]$. In Theorem 8 we show that under slightly weaker conditions $G \in O P^{\circ}$. Note that $O B^{\circ} \subseteq O P^{\circ}$ [2, p. 299].

THEOREM 8. If $\beta>0,|G|<1-\beta$ on $[a, b], \int_{a}^{b} G$ exists and $\int_{a}^{b} G^{2}$ exists, then $G \in O P^{\circ}$ on $[a, b]$.

Proof. It follows from Theorems 4 and 5 that $\int_{a}^{b} \ln (1+G)$ exists. Thus, since $\ln (1+G)$ is bounded on $[a, b]$, it follows from Lemma 3 that there 
exists a subdivision $D$ of $[a, b]$ and a number $B$ such that if $\left\{x_{a}\right\}_{q=0}^{n}$ is a refinement of $D$ and $0<u \leqq v \leqq n$, then $\left|\sum_{q=u}^{v} \ln \left(1+G_{q}\right)\right|<B$. Hence, if $\left\{x_{q}\right\}_{q=0}^{n}$ is a refinement of $D$ and $0<u \leqq v \leqq n$, then

Therefore, $G \in O P^{\circ}$ on $[a, b]$.

$$
\begin{aligned}
\left|\prod_{q=u}^{v}\left(1+G_{q}\right)\right| & =\left|\exp \sum_{q=u}^{v} \ln \left(1+G_{q}\right)\right| \\
& \leqq \exp \left|\sum_{q=u}^{v} \ln \left(1+G_{q}\right)\right|<e^{B} .
\end{aligned}
$$

THEOREM 9. If $\prod_{a}^{b}(1+G)$ exists and is not zero and $\int_{a}^{b} G$ exists, then $G \in O L^{\circ}$ on $[a, b]$.

Proof. Let $p \in(a, b]$. Since $G$ is bounded on $[a, b]$ (Theorem 6), it follows from Lemmas 1 and 2 that

(1) $\lim _{x, y \rightarrow p^{-}}[G(x, y)+G(y, p)-G(x, p)]=0$, and

(2) $\lim _{x, y \rightarrow p^{-}}[G(x, p)-G(x, y)-G(y, p)-G(x, y) G(y, p)]=0$.

Hence,

(3) $\lim _{x, y \rightarrow p^{-}} G(x, y) G(y, p)=0$.

Suppose $\lim _{y \rightarrow p^{-}} G(y, p)$ does not exist; then there exist a sequence $\left\{x_{q}\right\}_{q=1}^{\infty}$ converging to $p$ and a positive number $\varepsilon$ such that

(4) $x_{q-1}<x_{q}<p$, and

(5) $\left|G\left(x_{q-1}, p\right)-G\left(x_{q}, p\right)\right|>\varepsilon$.

There exists a subsequence $\left\{x_{q_{i}}\right\}_{i=1}^{\infty}$ of $\left\{x_{q}\right\}_{q=1}^{\infty}$ such that $\left\{G\left(x_{q_{i}}, p\right)\right\}_{i=1}^{\infty}$ is bounded away from zero. Hence, it follows from (3) that

Thus,

$$
\lim _{i \rightarrow \infty} G\left(x_{q_{i}-1}, x_{q_{i}}\right)=0 \text {. }
$$

$$
\lim _{x . y \rightarrow p^{-}}[G(x, y)+G(y, p)-G(x, p)] \neq 0 .
$$

Since this contradicts $(1), \lim _{y \rightarrow p^{-}} G(y, p)$ exists. It now follows from (1) that $\lim _{x, y \rightarrow p^{-}} G(x, y)=0$.

The existence of the right limits follows in a similar manner. Therefore, $G \in O L^{\circ}$ on $[a, b]$.

\section{BIBLIOGRAPHY}

1. W. P. Davis and J. A. Chatfield, Concerning product integrals and exponentials, Proc. Amer. Math. Soc. 25 (1970), 743-747. MR 42 \#1970.

2. B. W. Helton, Integral equations and product integrals, Pacific J. Math. 16 (1966), 297-322. MR 32 \#6167.

3. - A product integral representation for a Gronwall inequality, Proc. Amer. Math. Soc. 23 (1969), 493-500. MR 40 \#1562.

4. A. Kolmogoroff, Untersuchungen über den Integralbegriff, Math. Ann. 103 (1930), 654-696.

Department of Mathematics, Arizona State University, Tempe, Arizona 85281 